\title{
Laser Scanning Cytometry for the Pharmaceutical Industry.
}

\author{
E. Luther
}

CompuCyte Corporation, Cambridge, MA 02139

In analysis applications for the pharmaceutical industry, there is a parallel tradeoff betweenhigh throughput and high information content. High throughput is necessary for screening applications where libraries need to be screened for activity against a target typically using highly automated instruments that measure a single feature of the sample, and are especially well suited for detecting interactions between molecules in solution. High content analysisinvolves both having many detectors as well as extracting many features for each detector, and is applied typically to cellular models. High content analysis is necessary to effectively detect interactions that may affect only a subset of the cells in the sample (heterogeneous analysis), or to measure the effect of an agent on the distribution of constituents within a cell (sub-cellular analysis), or to study the relations amongst cells in a heterogeneous population.

In the pharmaceutical industry, high content analysis is particularly effective in lead confirmation studies, where it can help to answer such questions as: are candidate molecules reaching their intended targets (efficacy)?; is the molecule having an effect on the target?; does the molecule elicit the expected response in the cellular target? Does the response occur in the expected regions within the cell?

Toxicology is another well-suited application. Cell death, particularly in the apoptotic mode, is a complex series of events following multiple pathways that start with a signaling event and progress to physiological and morphological changes in the cells. High content analysis permits pinpointing not only if a compound is toxic to the cell, but at which stage and by what pathway it is having an effect.

Instruments are becoming commercially available with high content cellular analysis capability at relatively high throughput sample rates. These instruments fall into two classes: camera based systems and laser scanning systems. In camera based systems, a real image of the specimen is focused onto the detection surface of a high sensitivity digital camera. In laser scanning systems no real image is formed, but instead measurements of the output of photomultiplier tube and photodiode detectors are sampled at rates that are synchronized with a scanning laser beam and movements of an automated high-resolution microscope stage. The resulting sets of computer memory arrays are analogous to digital images, but are not congruent with them. In both systems, image processing techniques are applied that segment events of interest and calculate values for multiple features of interest.

CompuCyte Corporation (Cambridge MA) pioneered the application of high content analysis using laser scanning technology with the introduction of the LSC® Laser Scanning Cytometer $(1,2)$. After introduction, a number of technical advances were made in the use of the laser scan memory arrays as images in their own right. These advancements led to the development of an inverted format LSC designed for automated analysis of samples in microtiter wells where the microscope viewing components are eliminated. Cytometric analysis and high quality imaging is possible without microscope light sources, cameras, or oculars. Movable filter wheels and cubes are not needed, nor are optical path changing prisms or mirrors. Thus, with the simplified optical system, 
less time is spent on the mechanics of obtaining cellular images, and in fact they are obtained simultaneously with the fluorescence population data. As the purpose of the visual images is verification of the automated analysis results, it is potentially more informative to use the same optical system for image acquisition that was used for the population analysis.

[1] L.A.Kamentsky et al. Slide based laser Scanning Cytometry. Acta Cytologica. 1997: 41(1):123-143

[2] E. Luther and L.A. Kamentsky, Proceedings Microscopy and Microanalysis 1997, 235-236.

Laser Scanning Cytometry allows stoichiometric analysis. In this example, $\mathrm{CHO}$ cells were incubated for 48 hours with cytochalasin B. Population data resolves the cell cycle as either single parameter histograms or two parameter dot plots.

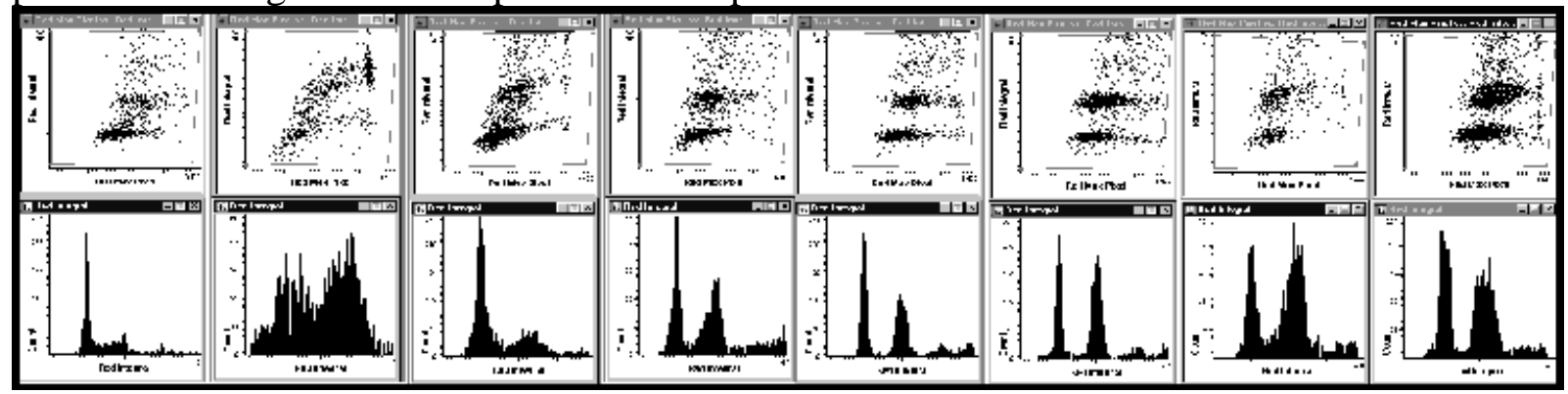

Laser Scatter Imaging allows detection of morphological changes. In this example, $\mathrm{CHO}$ cells were treated with increasing doses of colchicine. Changes in the morphology and growth habit of the cells are visible.

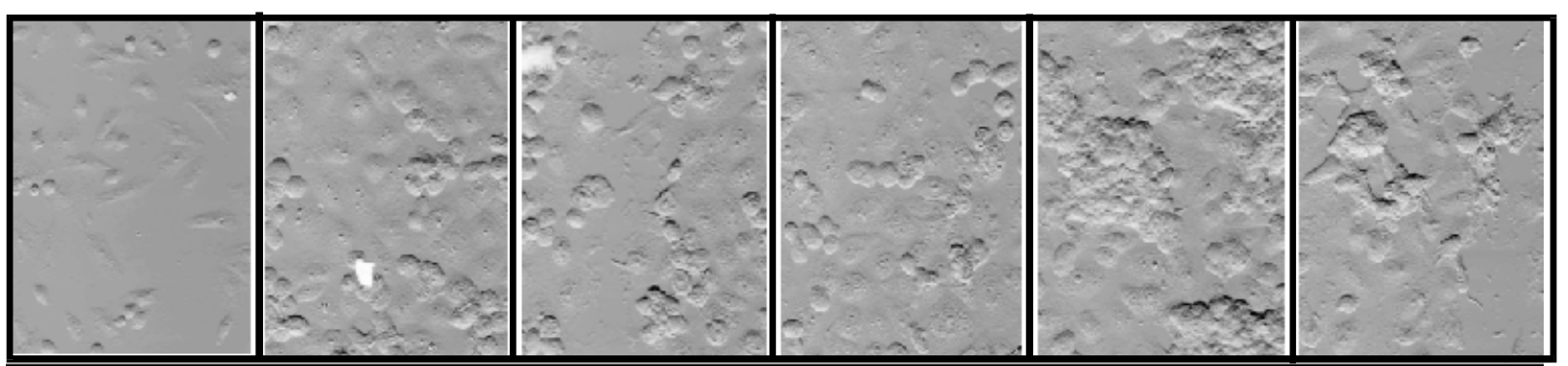

Laser Scanning cytometry unites population analysis with visual verification. Here, HeLa cells were stained for DNA content (red) and tubulin (green). Automatic acquisition of galleries of images from regions thought to be $\mathrm{G} 2$ and mitotic is confirmed by the cell morphology.
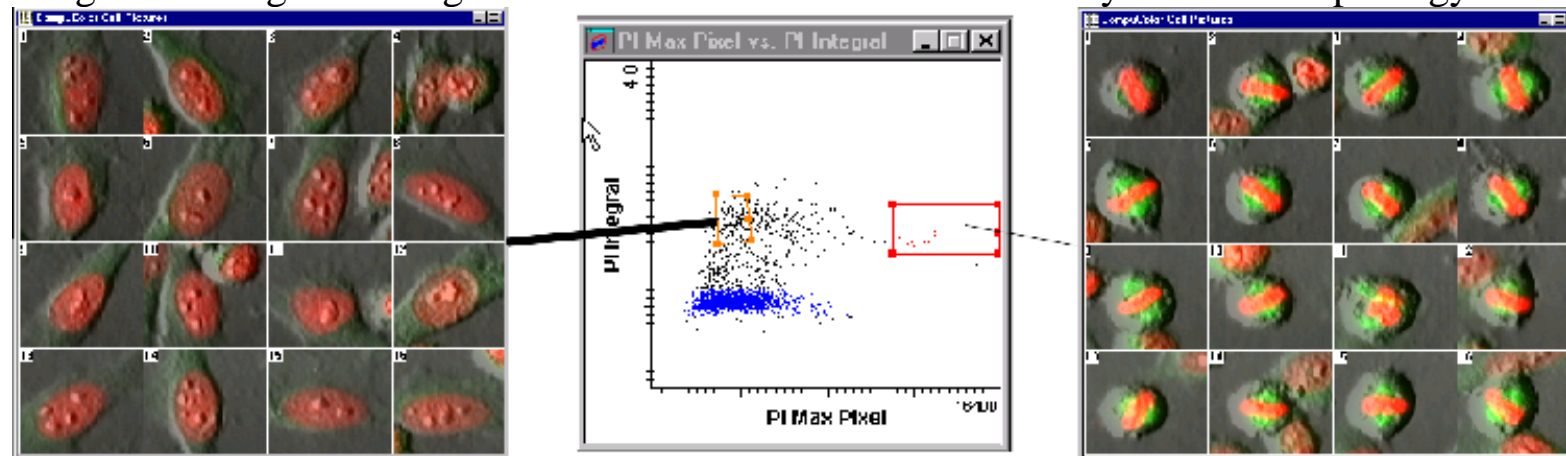